\title{
Stationary turbulent dynamo as spontaneous symmetry breaking
}

\author{
Hnatich M. \\ Institute of Experimental Physics of Slovak Academy of Sciences, \\ Watsonova 47, 04001 Košice, Czecho-Slovakia
}

\section{INTRODUCTION}

The large-scale magnetic field generation by the turbulent motion energy, known as turbulent dynamo [1], is perspective candidate to explain the observed stationary magnetic fields of cosmic objects.

It is well known that in magneto-hydrodynamic equations which describe reflectional non-symmetric (gyrotropic) conductive turbulent fluid some instabilities occure [2]. These instabilities lead to increasing magnetic fluctuation and to large-scale stationary magnetic field generation. However, to find the established magnetic field is a difficult problem because of the strong nonlinearities in MHD equations. Thus studies devoted to this problem usually consider non-stationary turbulent dynamo, i.e. they investigate the initial stage of large-scale magnetic field development on the basis of linearised MHD equations.

In contrary to traditional approaches, the quantum - field formalism $[4,5]$ is suitable to describe the final steady regime. In paper [3] this formalism has been used to treat the fully developed isotropic magneto-turbulence of gyrotropic incompessible fluid. The stationary turbulent dynamo has been explained by the spontaneous symmetry breaking mechanism widely employed in quantum theory.

\section{MODEL}

The stochastic MHD is governed by equations [6]:

$$
\begin{aligned}
\partial_{t} \mathbf{v} & =\nu \Delta \mathbf{v}-(\nabla \mathbf{v}) \mathbf{v}+(\nabla \mathbf{b}) \mathbf{b}-\nabla p+\mathbf{f} \\
\partial_{t} \mathbf{b} & =u \nu \Delta \mathbf{b}-(\nabla \mathbf{v}) \mathbf{b}+(\nabla \mathbf{b}) \mathbf{v}
\end{aligned}
$$

Here $\mathbf{v}(\mathbf{x}, t)$ denotes the transverse (incompressibility) velocity pulsations, $\mathbf{b}(\mathbf{x}, t)$ stand for the magnetic field fluctuations measured in Alfvén velocity units, $\nu$ is the molecular viscosity coefficient, $u=\nu^{\prime} / \nu\left(\nu^{\prime}\right.$ - magnetic 
diffusivity) is the inverse magnetic Prandtl number. The external random force $\mathbf{f}$ simulates the stochasticity of the problem, i.e. the interaction of the velocity pulsations with the mean flow. This force has Gaussian distribution $P(f)=\exp \left(-\frac{1}{2} f_{j} D_{j s} f_{s}\right)$ with given pair noise correlator

$$
<f_{j}^{v}(\mathbf{k}) f_{s}^{v}(-\mathbf{k})>\equiv D_{j s}(\mathbf{k})=d(k) \mathcal{Q}_{j s}
$$

(in wave vectors $\mathbf{k}$ and frequency $\omega$ representation, $k \equiv|\mathbf{k}|$ ). Choosing $d(k)$ one specifies the form of the energy pumping into the turbulent system which is necessary to compensate for dissipation losses. There is universally acknowledged choice of $d(k)$ [4] which corresponds to the energy pumping from the largest-scale eddies (or, equivalently, from the region of the small wave vectors $\mathbf{k}$ ). In gyrotropic MHD the transversal tensor $\mathcal{Q}_{j s}$ is of general form: $\mathcal{Q}_{j s}=P_{j s}+i \lambda \varepsilon_{j s l} k_{l} / k$, where $P_{j s}=\delta_{j s}-k_{j} k_{s} / k^{2}$. The antisymmetric tensor $\varepsilon$ models the reflectional symmetry violation (gyrotropy), $\lambda$ is the gyrotropy coefficient $(|\lambda| \leq 1)$.

In the field formulation the problem (1) is described by the action $S$ of the fields $\mathbf{v}, \mathbf{b}$ and some auxiliary transversal fields $\mathbf{v}^{\prime}, \mathbf{b}^{\prime}[6]$ :

$$
\begin{aligned}
S=\frac{1}{2} \mathbf{v} D \mathbf{v} & +\mathbf{v}^{\prime}\left[-\partial_{t} \mathbf{v}+\nu \Delta \mathbf{v}-(\nabla \mathbf{v}) \mathbf{v}+(\nabla \mathbf{b}) \mathbf{b}\right] \\
& +\mathbf{b}^{\prime}\left[-\partial_{t} \mathbf{b}+u \nu \Delta \mathbf{b}-(\nabla \mathbf{v}) \mathbf{b}+(\nabla \mathbf{b}) \mathbf{v}\right]
\end{aligned}
$$

The integration over the space-time variables and the trace over the vector indexes are implied.

The various Green (correlation and response) functions of velocity pulsations $\mathbf{v}$ and magnetic fluctuations $\mathbf{b}$, which we are interested in, are usual functional averages of corresponding fields with a weight $\exp S$. For example, pair velocity correlation function $<\mathbf{v v}>$ and magnetic response function $\left\langle\mathbf{b b}^{\prime}\right\rangle$ are determined as:

$<\mathbf{v v}>=\int d \mathbf{v} d \mathbf{b} d \mathbf{v}^{\prime} d \mathbf{b}^{\prime} \mathbf{v} \mathbf{v} \exp S, \quad<\mathbf{b}^{\prime}>=\int d \mathbf{v} d \mathbf{b} d \mathbf{v}^{\prime} d \mathbf{b}^{\prime} \mathbf{b} \mathbf{b}^{\prime} \exp S$.

These functions may be calculated by Feynman diagramatic technique. The main problem is, as usually in field theory, to eliminate singularities that are present in the perturbation theory diagrams for the correlation functions. For the reflectionary symmetric (non-gyrotropic) MHD this problem has been solved in paper [6]. Using the renormalization group methods the existence of infrared stable scaling regime has been demonstrated $[6,7]$. In this regime the inverse Prandtl number $u$ has some definite value.

But the additional singularity appears if the gyrotropy was included. This singularity causes that the magnetic responce function $\left\langle\mathbf{b b}^{\prime}\right\rangle$ increases exponentially with the time in the range of the small wave 
numbers $\mathbf{k}$. Thus the system becomes unstable, because the magnetic responce function must be retarded, and at the same time, it must ensure the damping of all perturbations. Note that this condition is fulfilled without nonlinearities in (1):

$$
<\mathbf{b}_{j}(\mathbf{k}, t) \mathbf{b}_{s}^{\prime}(-\mathbf{k}, 0)>=\theta(t) e^{-u \nu k^{2} t} P_{j s} .
$$

Here $\theta$ is the usual step function (equal to zero for $t<0$ and $\theta=1$ for $t>0$ ). The included nonlinearities lead to the appereance of an unstable term (further called a rotor term). In the first (one-loop) approximation it yields:

$$
\begin{aligned}
\left\langle\mathbf{b}_{j}(\mathbf{k}, t) \mathbf{b}_{s}^{\prime}(-\mathbf{k}, 0)\right\rangle & =\theta(t) e^{-u \nu k^{2} t}\left[P_{j s} \cosh a k t+i \varepsilon_{j s l} k_{l} / k \sinh a k t\right]+ \\
& + \text { stable terms. }
\end{aligned}
$$

Here constant $a$ is proportional to the gyrotropy parameter $\lambda$ and to the ultraviolet cutoff $k_{d} \approx r_{d}^{-1}$, where $r_{d}$ is the turbulent dissipation length. It is clear that $\langle\mathbf{b} \mathbf{b}\rangle \rightarrow \infty$ as $t \rightarrow \infty$ in range of small $\mathbf{k}$.

The instability problem may be solved on the basis of the spontaneous symmetry breaking mechanism. Briefly, this mechanism can be described as follows. The 'normal state' of the turbulent gyrotropic conductive fluid with zero mean value of the magnetic field is unstable, being stabilised by the spontaneous appearance of the space-uniform mean magnetic field $\mathbf{B} \equiv<\mathbf{b}>\neq 0$ just in the same way as a ferromagnetic below Curie temperature $T_{c}$ is stabilized by the appearance of spontaneous magnetisation. The value of the appeared field is determined by the system stability condition but, unlike the case in standart models, this condition is not reduced to a simple requirement, e.g. to the minimum free energy. In this case the assumption that $\mathbf{B} \neq 0$ is fixed directly in the action (3) by the replacing $\mathbf{b} \rightarrow \mathbf{b}+\mathbf{B}$, which leads to new perturbation theory diagrams for correlation functions. The stability condition is equivalent to the requirement that the unstable rotor term in magnetic responce function should be removed. This turned out to be possible with a proper choice of the $\mathbf{B}$ value. The direction of $\mathbf{B}$ is not fixed in this case and its absolute value is

$$
|\mathrm{B}|=\frac{16}{3} \sqrt{\frac{u \rho}{\pi}} \nu k_{d}
$$

where $\rho$ is the fluid density.

Note that the turbulent correlation length (external turbulent scale) is set to infinity in model (3) thus formally the large-scale magnetic field becomes space-uniform. One wants to emphasize also (detailes see in $[6,3]$ ) 
that the dynamo mechanism is realised in some infrared scaling regime, where the inverse Prandtl number $u=1.393$ (in one-loop approximation).

Expecting that the Earth magnetic field is generated by turbulent dynamo one can estimate the quantities in the expression for spontaneous field (5), taking

the mean magnetic field of Earth

the viscosity coefficient

the mass density in Earth core

the inverse magnetic Prandtl number

$$
\begin{array}{r}
B=0.3 \div 0.5 \text { Gauss } \\
\nu=10^{-2} \div 5 \times 10^{-2} \mathrm{~cm}^{2} / \mathrm{sec} \\
\rho=10 \div 12 \mathrm{~g} / \mathrm{cm}^{3} \\
u=1.393
\end{array}
$$
length.

and then $r_{d} \equiv k_{d}^{-1}=0.6 \div 4.4 \mathrm{~cm}$ is obtained for the dissipation turbulent

Taking the external turbulent scale $r_{c} \sim 3 \times 10^{8} \mathrm{~cm}$ (the radius of the Earth core) and using the known relation $r_{c}=R \epsilon^{3 / 4} r_{d}$ one yields large values of Reynolds number $R e=10^{10} \div 10^{11}$ which are typical for the fully developed turbulence.

\section{MODIFICATION OF ALFVÉN WAVES}

After the instability elimination demonstrated above the "exotic" term remains in $\left\langle\mathbf{b b}^{\prime}\right\rangle$ :

$$
<\mathbf{b}_{j}(\mathbf{k}, t) \mathbf{b}_{s}^{\prime}(-\mathbf{k}, 0)>=\theta(t) e^{-u \nu k^{2} t}[P_{j s}+\underbrace{i g \varepsilon_{j l m} k_{l} k_{m} B_{m} B_{s}}_{\text {"exotic"term }} t]+\ldots,
$$

where $\mathbf{e} \equiv \mathbf{B} /|\mathbf{B}|$ and the constant $g$ is proportional to the gyrotropy parameter $\lambda$ and to the mean magnetic field $|\mathbf{B}|$. Its physical sence can be clarified by analysis of the linearised MHD equations.

The linearised MHD equations without dissipative terms with spontaneous magnetic field $\mathbf{B} \neq 0$ and with exotic contribution are of the form:

$$
\begin{aligned}
\partial_{t} \mathbf{v} & =(\mathbf{B} \nabla) \mathbf{b}, \\
\partial_{t} \mathbf{b} & =(\mathbf{B} \nabla) \mathbf{v}+g[\nabla \times \mathbf{e}](\mathbf{b e}) .
\end{aligned}
$$

In order to find the solution an orthonormal basis of vectors $\mathbf{n}, \mathbf{l}, \mathbf{m}$ is chosen:

$$
\mathbf{n}=\mathbf{k} / k, \quad \mathbf{l}=(\mathbf{e}-\mathbf{n} \cos \alpha) / \sin \alpha, \quad \mathbf{m}=[\mathbf{n} \times \mathbf{e}] / \sin \alpha,
$$

where $\alpha$ is the angle between vectors $\mathbf{n}$ and e. The transversal fields $\mathbf{v}$, $\mathbf{b}$ are decomposite with respect to the orthonormal basis; $\mathbf{v}=v_{l} \mathbf{l}+v_{m} \mathbf{m}$, $\mathbf{b}=b_{l} \mathbf{l}+b_{m} \mathbf{m}$. The solution for scalar modes $v_{l}, v_{m}, b_{l}, b_{m}$ has the form:

$$
\begin{aligned}
& v_{l}=A_{1} \exp i(\mathbf{k x}-\omega t), \quad v_{m}=\left[-A_{2}+\frac{g_{1} A_{1}}{\omega}(1+i \omega t)\right] \exp i(\mathbf{k x}-\omega t), \\
& b_{l}=-A_{1} \exp i(\mathbf{k x}-\omega t), \quad b_{m}=\left[A_{2}-i g_{1} A_{1} t\right] \exp i(\mathbf{k x}-\omega t)
\end{aligned}
$$


Here $A_{1}, A_{2}$ are arbitrary constant amplitudes, $g_{1}=g \sin \alpha$ and $\omega=\mathrm{Bk}$.

It seems that wave modes $m$ contain contributions that increase linearly with time. The inclusion of dissipative terms into linearised equations (8) results in exponential damping $\exp (-\beta t)$, which suppresses the linear growth for arbitrary small $\beta \sim \nu k^{2}$.

Therefore, the exotic contribution, which necessarily appears in the dynamo regime, does not generate instability but facilitates the formation of specific long-lived pulses of order $t \exp (-\beta t)$ in Alfvén waves polarized at right angle to the spontaneous field.

\section{References}

[1] Vainshtein S.I., Zeldovich Ya.B., Ruzmaikin A. Turbulentnoye dynamo $v$ astrofizike, Nauka (Moscow), (1980), 351 pp.

[2] Krause F., Rädler K.-H. Mean-field magnetohydrodynamics and dynamo theory, Pergamon, Oxford OX3 0BW (1980), 271 pp.

[3] Adzhemyan L.Ts., Vasilev A.N., Hnatich M. Teor, i mat. fizika (Russian J.) 72 (1987) 369.

[4] De Dominicis C., Martin P.C. Phys. Rev. A 19 (1979) 419.

[5] Adzhemyan L.Ts., Vasilev A.N., Pis'mak Ju.M. Teor. $i$ mat. fizika (Russian J.) 57 (1983) 268.

[6] Adzhemyan L.Ts., Vasilev A.N., Hnatich M. Teor. i mat. fizika (Russian J.) 64 (1985) 196.

[7] Fournier J.D., Sulem P.L., Pouquet A. Phys. Rev. A 15 (1982) 1393. 